\title{
Wireless Multi-Sensor Gas Platform for Environmental Monitoring
}

\author{
1, * Denis Spirjakin, ${ }^{1}$ Alexander Baranov, ${ }^{2}$ Alexey Karelin, ${ }^{3}$ Andrey Somov \\ 1 "MATI" -Russian State Technological University, Moscow, Russia \\ *e-mail: denis.spirjakin@gmail.com \\ 2 “STC MGS” Lyubertsy, Moscow Region, Russia \\ ${ }^{3}$ CREATE-NET, Trento, Italy
}

\begin{abstract}
Air quality control is a monitoring task of high priority in the scope of Smart City and Smart Home applications. In fact, a number of sensors are required to successfully detect the leaks of various gases and their different concentrations. In this work, we present an autonomous multisensory wireless platform which includes both analog and digital gas sensors for environmental monitoring. The platform includes four measurement circuits with sensors of different types: one for analog catalytic/semiconductor gas sensor, two for analog electrochemical gas sensors and one for a gas sensor with digital data transmission interface. The platform ensures low power consumption and can operate either as a separate monitoring sensor node or as a part of wireless sensor network.
\end{abstract}

Keywords - multisensory wireless platform; analog and digital gas sensors, power management, environmental monitoring

\section{INTRODUCTION}

The paradigm of Wireless Sensor Networks (WSN) [1] is considered as a pillar technology of the forthcoming era of Internet of Things (IoT) [2] where the smart-x applications such as Smart City [3] and Smart Home [4] play a major role. Air quality monitoring is among the key tasks in the context of these applications. Indeed, it is a problem of high priority to ensure the comfort and safe environment for people to improve their quality of life [5].

However, a number of leaks of various hazardous gases, e.g. hydrocarbons, carbon monoxide, carbon dioxide, oxygen, of different concentration may occur in a city/plant/house environment [6][7].

The WSNs consisting of tiny, autonomous sensing devices are widely used in monitoring and control applications [8][9] including gas detection one [10][11]. The WSNs can be deployed in a difficult to access area, cover a large territory, and guarantee continuous detection of combustible, toxic and explosive gases [12][13] as well as preventing the catastrophic consequences due to the activation of wireless actuators closing the gas valves, disconnecting the power supply and perform other functions [14].

To perform the secure detection of various gases of different leak concentration, heterogeneous sensor types have to be applied [15], e.g. semiconductor, catalytic,

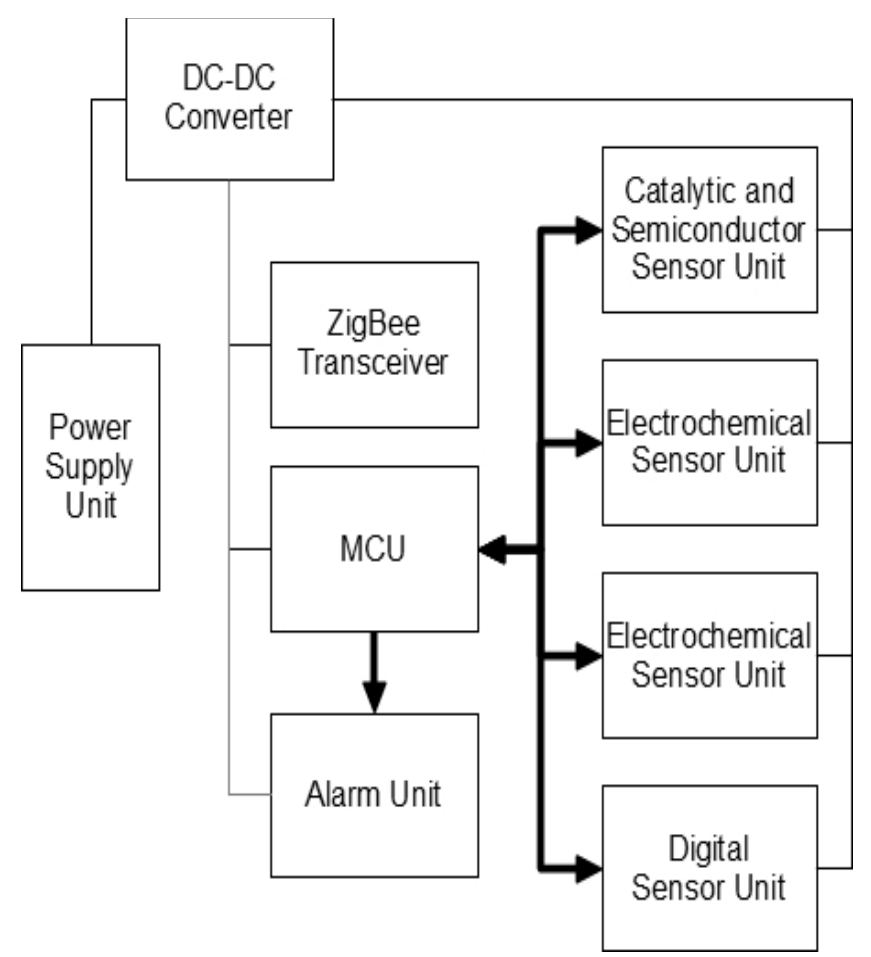

Fig. 1. Block diagram of wireless multisensor node.

electrochemical, optical. This imposes a multi-sensor requirement for a sensing platform.

A number of WSN platforms for gas sensing have been proposed recently. Most of them are based on a single gas sensor [7][14][10]. The platforms based on the multi-sensor technology are still fragmented [15] and require further research and evaluation of this approach.

In this paper, we present an autonomous multi-sensor wireless platform for various gases leaks detection. The platform supports the analog and digital gas sensors for environmental monitoring and emergency control. The proposed sensor node platform is optimized for low power consumption operation and can function as a separate sensor node or as a part of IEEE 802.15.4 ZigBee based WSN. 
The paper is organized as follows: we first overview the platform in Section II. In Section III and Section IV we describe sensing circuits for catalytic/semiconductor and electrochemical sensors, respectively. Digital sensing circuit is discussed in Section V. The evaluation of power consumption of sensor node is presented in Section VI. Finally, we provide concluding remarks in Section VII.

\section{SYSTEM OVERVIEW}

The block diagram of the wireless gas sensor node is presented in Fig. 1. The sensor node is based on an ATxmega32A4 Microcontroller Unit (MCU) and use an ETRX3 communication module. The selection of the MCU is mainly driven by the following requirements: low power consumption, on-chip temperature sensor, and precise ADC integrated in the MCU.

The wireless communication unit employs the low power ETRX3 wireless modem supporting IEEE 802.15.4 standard (ZigBee specification) and transmitting in unlicensed $2.4 \mathrm{GHz}$ ISM band. The modem has an integrated chip antenna used in this design (up to $25 \mathrm{~m}$ ) and a connector for an external antenna to enable a boost mode allowing data transmission for up to $350 \mathrm{~m}$. The modem has a number of self-x features enabling, e.g. WSN self-configuration and self-diagnostics which significantly reduce debugging and deployment time.

Voltage conversion from batteries is performed by a DCDC converter TPS63060 to provide maximum efficiency. The device generates stable output voltage of $3.3 \mathrm{~V}$ from $2.5 \mathrm{~V}$ to $12 \mathrm{~V}$ on its input.

Alarm unit includes several light emitting diodes and a sound alarm. The alarm unit is essential for notifying a user about dangerous gas concentration in the environment or batteries discharge. In this case, the node operates as a local sensor-actuator system without connecting to a WSN.

The multi-sensor platform includes four sensing circuits to support the sensors of different types: one circuit for a catalytic or semiconductor gas sensor, two circuits for the electrochemical gas sensors and one for a gas sensor with a digital interface.

In this work, the multisensory platform is equipped with the following sensors: a semiconductor sensor for organic material pyrolysis detection, catalytic sensor for $\mathrm{CH}_{4}$ (methane) detection, electrochemical sensors with an analog output for $\mathrm{CO}$ (carbon monoxide) and $\mathrm{H}_{2} \mathrm{~S}$ (hydrogen sulphide) detection, and optical sensor with a digital interface for the detection of $\mathrm{CH}_{4}$ of high concentration.

The catalytic sensor is used to conduct the $\mathrm{CH}_{4}$ measurements in the range of Lower Explosive Limit (LEL) concentration. The semiconductor sensor has high sensitivity and selectivity to detect the products of pyrolysis $\left(\mathrm{H}_{2}\right.$ and $\left.\mathrm{CO}\right)$ [26]. Both the semiconductor sensor for pyrolysis detection [22] and catalytic sensor for $\mathrm{CH}_{4}$ detection [23] are fabricated on an aluminum-oxide membrane using micromachining technology. This technology ensures the low power consumption of the sensors, i.e. around $70 \mathrm{~mW}$ in continuous mode. For the detection of $\mathrm{CO}$ and $\mathrm{H}_{2} \mathrm{~S}$ the electrochemical sensors operating in amperometric mode [24] NAP-505 and
NE4-H2S by Nemoto, respectively, are used. As a sensor with a digital interface, we use MIPEX infrared gas sensor which has the measurement range from Upper Explosive Limit (UEL) up to $100 \%$ vol. of $\mathrm{CH}_{4}$ [25].

\section{Sensing Circuit For CATALYtiC AND SEMiCONDUCTOR SENSORS}

The sensing circuit based on the catalytic and semiconductor sensors is presented in Fig. 2. These sensors are combined in one circuit because both of them include heaters and their control schematics is the same.

To heat up the sensor, the pulse-width modulated signal is used. Pulse-Width Modulation (PWM) is frequently used to heat sensors up to working temperature [10][27]. These pulses are applied to a sensor and heat it up. Output signal of the heater is the voltage on resistive divider which is formed by the heater itself and reference resistor $R_{21}$. The output signal from this divider is applied to the amplifier's input. Another input of the amplifier is used to apply the reference voltage which is subtracted from the output signal to exclude a constant component of the signal.

The response of sensitive layer of semiconductor sensor is the voltage on the resistive divider with range selection which is formed by the sensor sensitive layer itself and resistors $R_{23}$ and $R_{25}$. The output signals from the amplifier and semiconductor sensor sensitive layer are measured by the ADC of MCU.

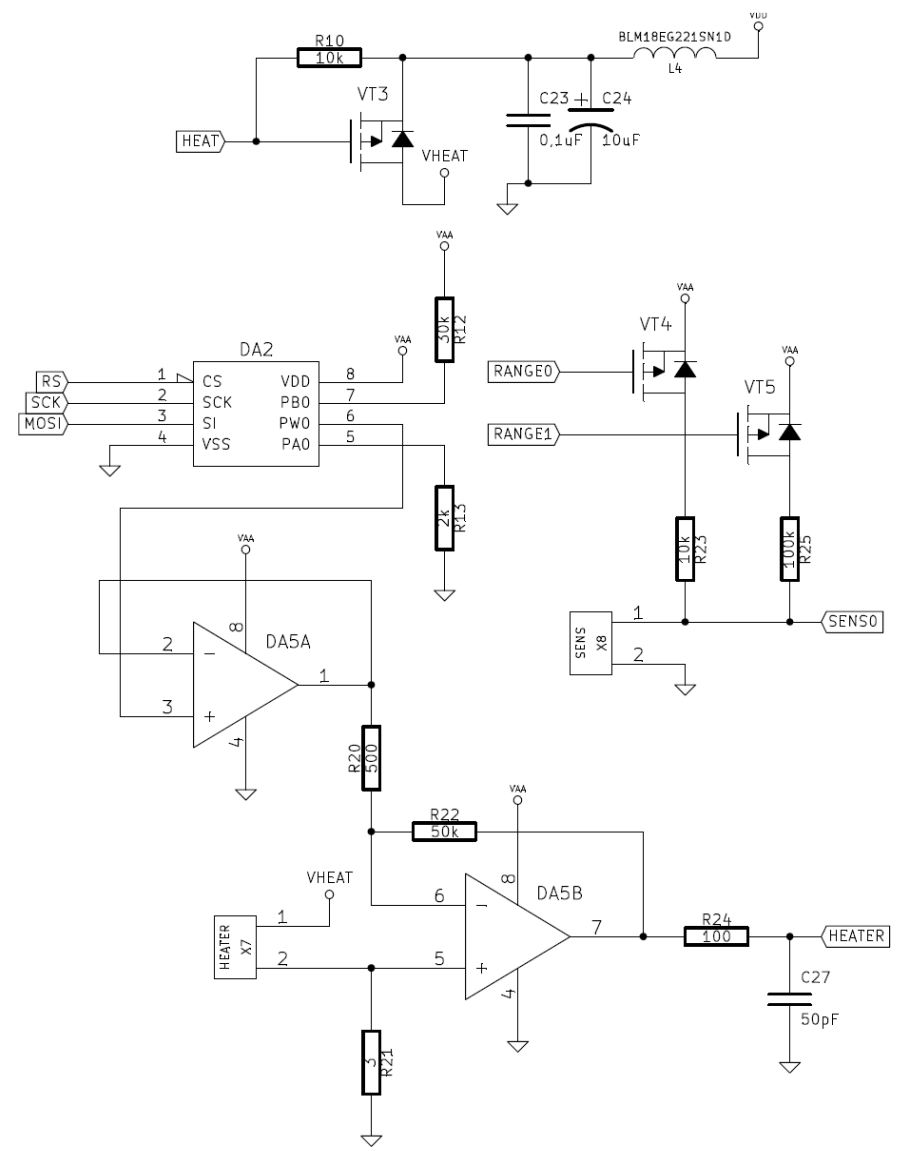

Fig. 2. Sensing circuit for the catalytic/semiconductor sensors. 


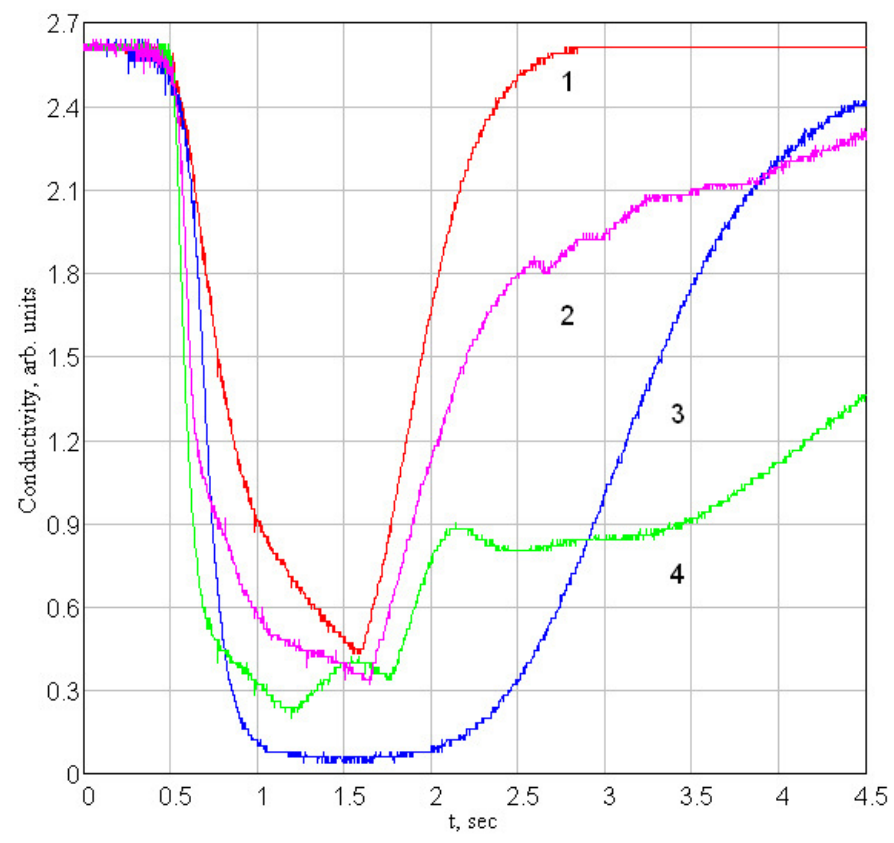

Fig. 3. Sensor's response to various gases at $\mathrm{T}=500{ }^{\circ} \mathrm{C}$ : $(1)-$ the air; $(2)-$ pyrolysis; (3) - $0.2 \% \mathrm{CH} 4$; (4) - alcohol fume.

Gas concentration measurements with semiconductor gas sensor are performed in the temperature scanning mode [10]. In this mode the sensor is heated up periodically and the measurements are performed in different temperature points.

Semiconductor gas sensor's response to various gases is presented in Fig. 3. As it shown in this figure, it is possible to select the right measuring points to exclude unnecessary influence of different gases.

Since the heating is performed periodically the power consumption of the sensor decreases.

Therefore, using this mode the sensor power consumption can be decreased which will result in the improved accuracy and selectivity of measurements.

The sensing circuit employing the catalytic sensor conducts the measurements using the multistage heating profile [17][18].

Sensing circuits, based on the catalytic sensors, use two sensors typically embedded in the Wheatstone circuit. It includes two resistors and two sensors, one active and one for the reference. During the measurements, most of the power goes into the sensor heating process (about $450{ }^{\circ} \mathrm{C}$ for methane detection), required to perform the measurement. The power consumption in this case is around $200 \mathrm{~mW}$ which is a huge number for the WSN applications. Excluding the reference sensor it is possible to decrease the power consumption. Another disadvantage of this circuit is the need of compensation for the atmosphere humidity and temperature. This compensation can be successfully accomplished using the multistage heating profile [17]. The response of the catalytic sensor in this case is the difference between the heater voltage in two different points. Output residual signal values for different methane concentrations are presented in Fig. 4.

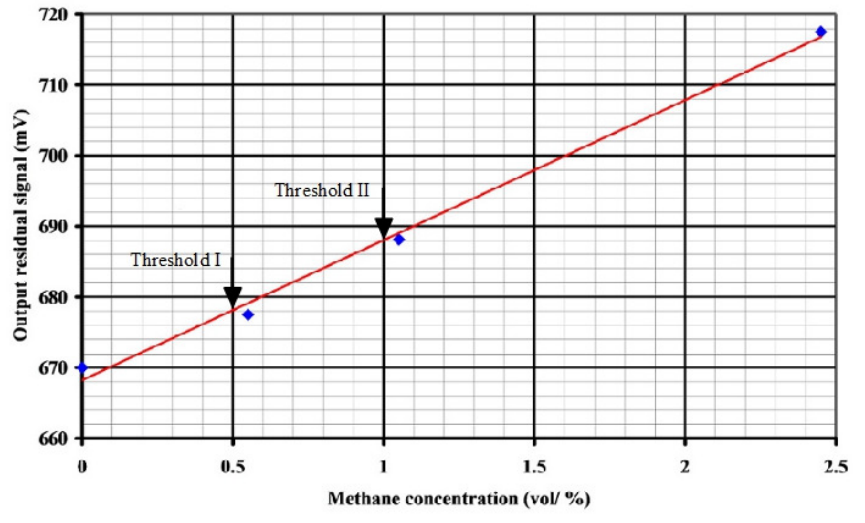

Fig. 4. Output residual signal values for different methane concentrations for catalytic sensor in multistage heating mode.

During the methane measurement, the sensor operates as a two-threshold device. If the methane concentration is less than $0.15 \%$ vol., the obtained values are stored in the memory of MCU, sending data wirelessly is not performed. If the concentration is less than $0.5 \%$ vol. methane sensor provides the light and sound alarm and sends the alarm messages if the predetermined threshold concentration is exceeded. If the methane concentration exceeds $1 \%$ vol., the alarm sounds, accompanied by light signaling and on the network coordinator or control device is sent to the alarm about the dangerous concentration of methane in the atmosphere. Also, the sensor can issue a command to the actuator, for example, a wireless gas valve. The mentioned thresholds can be changed by the program in the MCU.

\section{SENSING CirCUIT FOR ElECTROCHEMICAL SENSORS}

The measurement circuit for the electrochemical sensor is presented in Fig. 5. The circuit consists of two parts. The first part guarantees the stable functioning of the sensor. The second one conducts the measurements.

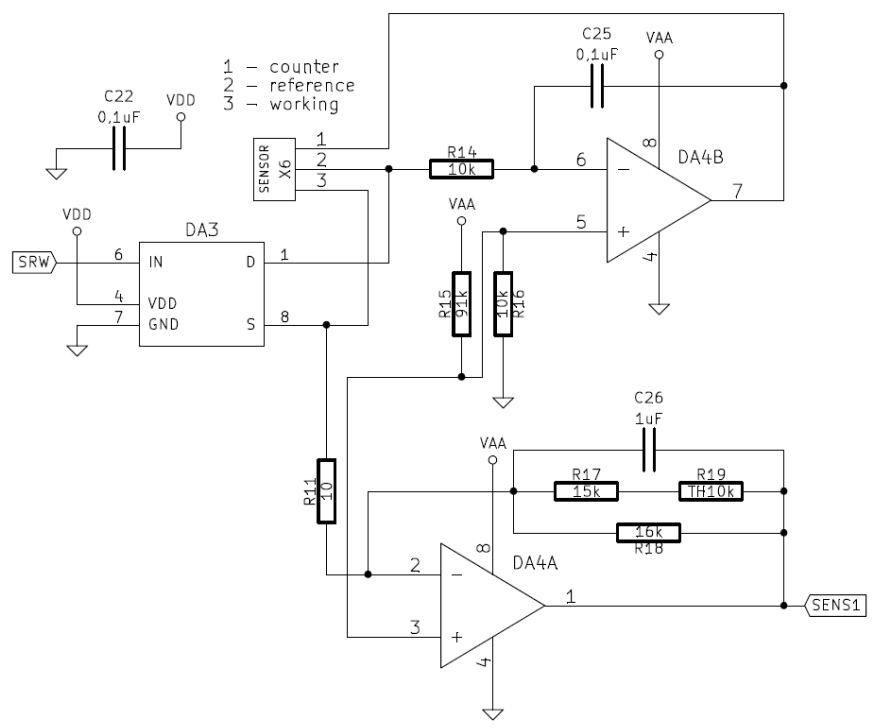

Fig. 5. Sensing circuit with the electrochemical sensor. 


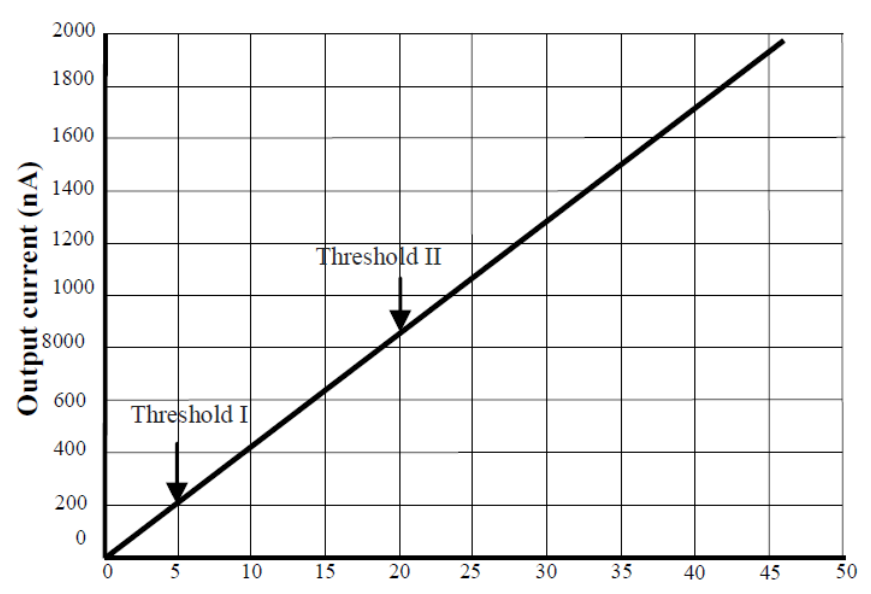

Gas concentration (ppm)

Fig. 6. Carbon monoxide electrochemical gas sensor response.

The reference electrode of the sensor maintains the healthy operation of the cell. Its electrochemical potential always remains constant and this is used to adjust the potential of the working electrode. This adjustment is performed by using the integrator which consists of amplifier $D A 4 B$, resistor $R_{14}$ and capacitor $C_{25}$.

The response of the sensor is the current between counter and working electrodes. The examples of sensor response which depends on the $\mathrm{CO}$ and $\mathrm{H}_{2} \mathrm{~S}$ concentration are shown in Fig. 6 and Fig. 7, respectively.

To get the measurement in a convenient voltage form it is necessary to convert the current response of the sensor to the voltage one. This conversion can be effectuated by the current sensing circuit based on the amplifier DA4A.

When the electrochemical sensor is used in periodic mode, the transient signals may appear right after the power is on and when the power is off. At normal operation, 1-2 minutes would be required for the output stabilization. To eliminate this effect it is necessary to maintain a short-circuit between the working electrode and the reference electrode when the power supply is off. In this case the output is stabilized within 10 seconds after the power is on [21].

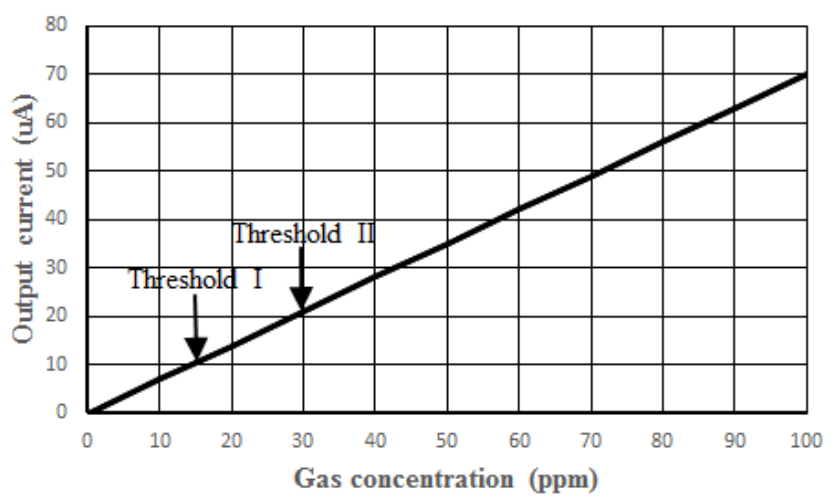

Fig. 7. Hydrogen sulfide electrochemical gas sensor response.
To connect the working and the reference electrodes together the MOSFET key DA3 is used.

For CO monitoring the sensor node is programmed to detect two thresholds (see Fig. 6), set to $T_{I}=5 \mathrm{mg} / \mathrm{m}^{3}$ and $T_{I I}=20 \mathrm{mg} / \mathrm{m} 3$, respectively. For $H_{2} S$ monitoring these values are $10 \mathrm{mg} / \mathrm{m}^{3}$ and $20 \mathrm{mg} / \mathrm{m}^{3}$. Threshold limit values for these gases are $20 \mathrm{mg} / \mathrm{m}^{3}$ for $C O$ and $10 \mathrm{mg} / \mathrm{m}^{3}$ for $H_{2} S$. All threshold values are stored in the MCU memory. The mentioned thresholds can also be changed within the program. If the detected concentration is less than $T_{I}$ - the sensor node goes to sleep mode; if the concentration is between $T_{I}$ and $T_{I I}-$ a local sound alarm announces about the increased $C O$ concentration; finally, if the concentration is higher than $T_{I I}-$ the node transmits an alarm message to an operator via the network coordinator. As a result, the operator can (de)activate an actuator, e.g., a gas valve, to avoid potentially dangerous situations.

\section{SEnsing CirCuit with DigitAl InTERFACE}

There are several commonly used digital interfaces for gas sensors: UART, SPI and $\mathrm{I}^{2} \mathrm{C}$. Since all of these interfaces are available in the MCU it is possible to combine all of them in one connector and have an opportunity to use almost every digital gas sensor. At the same time most of them use different protocols. Therefore, every sensor claims to support it in the node software.

Since digital sensors are often power hungry there is an option to switch them off. However, for the infrared sensors their stabilization time, i.e. wake up, takes up to two minutes in the worst case. If measurements are performed in a frequent periodic mode the sensors switching off is unreasonable.

In this work, we use MIPEX infrared gas sensor. To communicate the data it uses the UART interface. The sensing circuit for MIPEX sensor is presented in Fig. 8. The measurement range of methane for this sensor is up to $100 \%$. And the response time is less than 30 seconds.

\section{POWER CONSUMPTION}

Safety standards claim to perform gas sensors calibration every year. These requirements are provided in Russian Standard [19] and European Standard [20]. Therefore, it is reasonable to have autonomous lifetime of the sensor node no less than one year without the replacement of batteries.

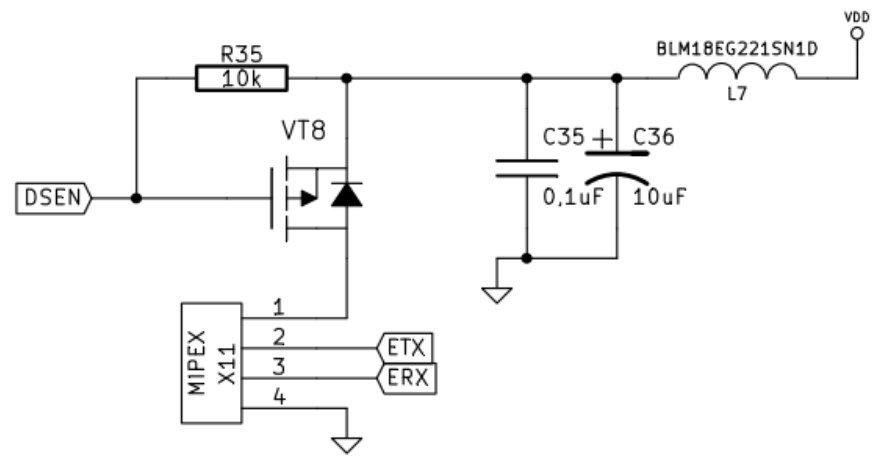

Fig. 8. Sensing circuit with the digital interface for MIPEX sensor. 


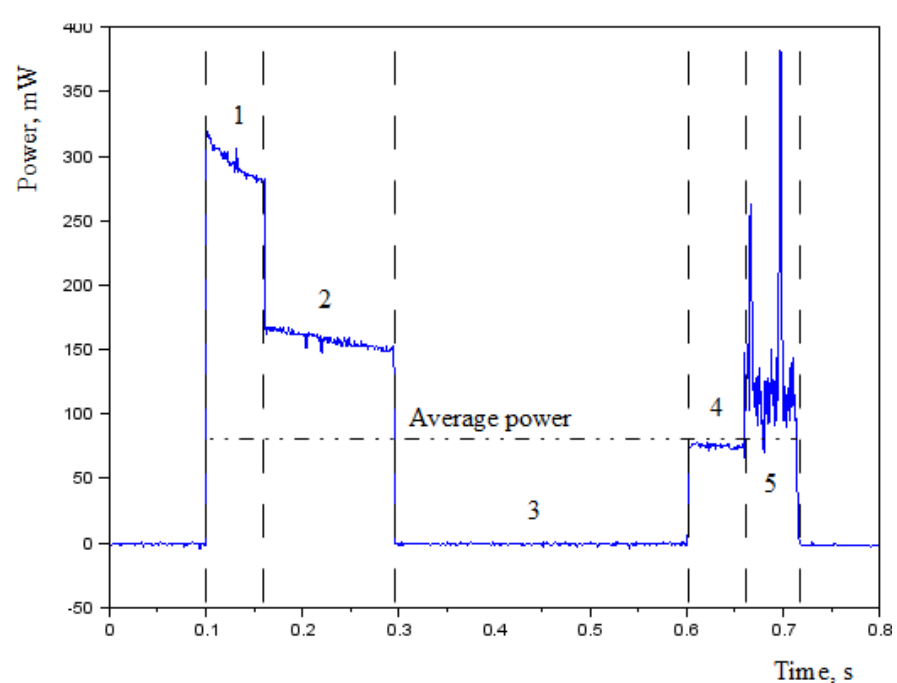

Fig. 9. The power consumption of the wireless multisensor node for gas detection: measurents (1-4) and data transmittion (5).

Capacity of a single cell lithium battery of $D$ type is typically $15000 \mathrm{mAh}$. Its voltage is $3.6 \mathrm{~V}$. Since there are 8760 hours a year, the average power consumption for one year sensor node lifetime is no more than $6 \mathrm{~mW}$.

In order to decrease power consumption, all circuits of the node are designed to perform measurements periodically. According to safety standards, sensor node response time should be less than 20 seconds. Therefore, two measurements every 20 seconds are sufficient to meet this requirement.

Measurements with all four sensors are made sequentially, one after another. First, heating of semiconductor or catalytic gas sensor starts. Since it does not take much time, the measurements with other sensors are performed during the heating process: (1) with the carbon monoxide electrochemical gas sensor, after that (2) with the hydrogen sulfide electrochemical gas sensor and then (3) the data from digital gas sensor is read. After these measurements, the heating process is brought to the end and measurements with semiconductor or catalytic gas sensor is performed. Finally, the device goes in sleep mode.

The power consumption of the node with catalytic gas sensor and two electrochemical sensors is presented in Fig. 9. It shows that the main power sink is the catalytic gas sensor (stages 1-4). Its average power consumption for multistage pulse is $81 \mathrm{~mW}$. This value includes power consumption for data transmission (stage 5). The average power consumption for one electrochemical sensor is about $5 \mathrm{uW}$ (that is in stage 1 of Fig. 9, but their contribution is too negligible to recognize it).

Since the power is consumed within only $0.7 \mathrm{~s}$, the average power consumption for overall period of $10 \mathrm{~s}$ is about 4.9 $\mathrm{mW}$.

To further decrease the power consumption, the node has to activate the wireless transmitter in the case of emergency situations: there is no point in data transmission after each measurement procedure. The average power consumption excluding data transmission is about $77 \mathrm{~mW}$. The average power consumption for overall period in this case is $4.6 \mathrm{~mW}$. This value guarantees one year of the node autonomous lifetime.

The semiconductor gas sensor is more power hungry because it takes longer time to heat it up. It consumes around $70 \mathrm{~mW}$ within $1.5 \mathrm{~s}$. It leads to the increase of power consumption for up to $5.25 \mathrm{~mW}$.

Digital gas sensor consumes $3 \mathrm{~mW}$ and takes long time for stabilization. The overall power consumption in combination with the catalytic sensor is $7.6 \mathrm{~mW}$ and in combination with the semiconductor gas sensor is $8.25 \mathrm{~mW}$. These values are more than $6 \mathrm{~mW}$ and in this case the autonomous lifetime of the node is less than one year.

Therefore, using all sensors at the same time leads to significant power consumption that makes it impossible to maintain the long lifetime of the sensor node. To increase the autonomous lifetime, some sensors should be used only for specific measurements from time to time.

Power consumption for the electronic devices used in this work is presented in Table I.

TABLE I. POWER CONSUMPTION OF ELECTRONIC DEVICES USED IN THIS WORK

\begin{tabular}{|l|c|}
\hline Device & Power consumption \\
\hline MCU & $1.88 \mathrm{~mW}$ \\
\hline Transceiver & $0.71 \mathrm{~mW}$ \\
\hline DC-DC Converter & $0.1 \mathrm{~mW}$ \\
\hline Catalytic gas sensor & $2.29 \mathrm{~mW}$ \\
\hline Semiconductor gas sensor & $5.25 \mathrm{~mW}$ \\
\hline Electrochemical gas sensor & $5 \mathrm{uW}$ \\
\hline Digital gas sensor & $3 \mathrm{~mW}$ \\
\hline
\end{tabular}

\section{CONCLUSION}

In this work, we have presented the autonomous wireless multi-sensor platform for environmental monitoring with a special focus on gas detection. This platform includes four sensing circuits for the sensors of different types: one for the analog catalytic or semiconductor gas sensor, two for the analog electrochemical gas sensors and one for a gas sensor with a digital data transmission interface.

Since the platform includes alarm facilities it can operate individually or as a part of IEEE 802.15.4 ZigBee standard WSN.

The measurements for this platform are performed periodically and can involve all sensors simultaneously or use only one or several of them. Due to this reason and special approaches for performing the measurements with the catalytic and semiconductor gas sensors, the low power consumption is achieved.

The average power consumption for combination of semiconductor, electrochemical and digital optical sensors is $8.25 \mathrm{~mW}$. This value can be further decreased by reducing the 
measurement duty cycle. The better values are obtained for combination with catalytic gas sensor instead of the semiconductor one. In this case, the average power consumption is about $7.6 \mathrm{~mW}$. The experimental results demonstrate that the proposed multi-sensor platform can ensure the long term autonomous monitoring.

\section{ACKNOWLEDGMENT}

This work was supported by the grant No. RFMEFI57714X0133 from the Ministry of Education and Science of Russian Federation.

\section{REFERENCES}

[1] V. C. Gungor, G. P. Hancke, "Industrial wireless sensor networks: challenges, design principles, and Technical approaches," IEEE Trans. Ind. Electron., vol. 56, no. 10, pp. 4258-4265, Oct. 2009.

[2] D. Miorandi, S. Sicari, F. De Pellegrini, I. Chlamtac, "Internet of things: vision, applications and research challenges", Ad Hoc Networks, vol. 10, no. 7, pp. 1497-1516, Sept. 2012,.

[3] A. Zanella, N. Bui, A. Castellani, L. Vangelista, M. Zorzi, "Internet of things for smart cities," IEEE Internet of Things Journal, vol.1, no.1, pp.22-32, Feb. 2014.

[4] D. Kelaidonis, A. Somov, V. Foteinos, G. Poulios, V. Stavroulaki, P. Vlacheas, P. Demestichas, A. Baranov, A.R. Biswas, R. Giaffreda, "Virtualization and cognitive management of real world objects in the internet of things," IEEE International Conference on Green Computing and Communications (GreenCom), pp.187-194, 20-23 Nov. 2012.

[5] V. Jelicic, M. Magno, D. Brunelli, G. Paci, L. Benini, "Context-adaptive multimodal wireless sensor network for energy-efficient gas monitoring," IEEE Sensors Journal, vol.13, no.1, pp.328-338, Jan. 2013.

[6] A.N.M. Karim, A.N. Nordin, S. Begum, "Technical and economic feasibility of sensor technology for health/environmental condition monitoring," Comprehensive Materials Processing, vol 13, pp. 499-514, 2014.

[7] M.I. Mead, O.A.M. Popoola, G.B. Stewart, P. Landshoff, M. Calleja, M. Hayes, J.J. Baldovi, M.W. McLeod, T.F. Hodgson, J. Dicks, A. Lewis, J. Cohen, R. Baron, J.R. Saffell, R.L. Jones, "The use of electrochemical sensors for monitoring urban air quality in low-cost, high-density networks," Atmospheric Environment, vol. 70, pp. 186-203, 2013.

[8] L. Mottola, G. P. Picco, M. Ceriotti, Ş. Gună, A. L. Murphy, "Not all wireless sensor networks are created equal: A comparative study on tunnels," ACM Trans. Sen. Netw. vol.7, no.2, article 15, 33 pages, Sept. 2010.

[9] D. Brunelli, I. Minakov, R. Passerone, M. Rossi, "POVOMON: an adhoc wireless sensor network for indoor environmental monitoring," IEEE Workshop on Environmental Energy and Structural Monitoring Systems (EESMS), pp.1-6, 17-18 Sept., 2014.

[10] A. Somov, R. Passerone, D. Spirjakin, M. Ivanov, I. Khromushin, A. Baranov, A. Savkin, "Combustible gases and early fire detection: an autonomous system for wireless sensor networks, 1st International Conference. on Energy-Efficient Computing and Networking 1st
International Conference on Energy-Efficient Computing and Networking (e-Energy), pp. 85-93, 13-15 April, 2010.

[11] Y.W. Kim, S.J. Lee, G.H. Kim, G.J. Jeon, "Wireless electronic nose network for realtime gas monitoring system," in Proc. ROSE, 2009, pp. 169-172.

[12] Li-Ling Hung, Yu-Wei Huang, Chun-Cheng Lin, "Temporal coverage mechanism for distinct quality of monitoring in wireless mobile sensor networks," Ad Hoc Networks, vol. 21, pp. 97-108, Oct. 2014,

[13] G. Owojaiye, Y. Sun, "Focal design issues affecting the deployment of wireless sensor networks for pipeline monitoring," Ad Hoc Networks, vol. 11, pp. 1237-1253, 2013.

[14] A. Somov, A. Baranov, and D. Spirjakin, "A wireless sensor-actuator system for hazardous gases detection and control," Sens. Actuators A, Phys., vol. 210, pp. 157-164, Apr. 2014.

[15] M. Hautefeuille, B. O'Flynn, F. H. Peters, C. O’Mahony, “Development of a microelectromechanical system (MEMS)-based multisensor platform for environmental monitoring," Micromachines, vol. 2, pp. 410-430, 2011.

[16] Online: http://book.itep.ru/4/41/zigbee.htm.

[17] A.Somov, A.Baranov, D.Spirjakin, R. Passerone, "Circuit design and power consumption analysis of wireless gas sensor nodes: One-sensor versus two-sensor approach", IEEE Sensors Journal, vol.14, pp.20562063, Apr. 2014.

[18] A. Somov, A. Baranov, A. Savkin, M. Ivanov, L. Calliari, R. Passerone, E. Karpov, A. Suchkov, Energy-aware gas sensing using wireless sensor networks,in: G.P. Picco, W. Heinzelmann (Eds.), Proceedings of the 9th European Conference on Wireless Sensor Networks (EWSN 2012), vol. 7158 of LectureNotes in Computer Science, February 15-17, Trento, Italy, Springer-Verlag,Berlin/Heidelberg, 2012, pp. 245-260.

[19] Standard GOST R EN 50194-1-2012, Signalizators for the detection of combustible gases in domestic premises, 2000.

[20] Standard EN 50194-2000, Electrical apparatus for the detection of combustible gases in domestic premises. Test methods and performance requirements, 2000.

[21] http://www.nemoto.eu/nap-505.html.

[22] A. A. Vasiliev, R. G. Pavelko, S. Yu. Gogish-Klushin, N. Samotaev et al., "Alumina MEMS platform for impulse semiconductor and IR optic gas sensors," Sensors and Actuators B: Chemical, vol. 132, no. 1, pp. 216-223, May 2008.

[23] E. E. Karpov, E. F. Karpov, A. Suchkov, S. Mironov, A. Baranov, V. Sleptsov, L.Calliari, "Energy efficient planar catalytic sensor for methane measurement,Sensors and Actuators", Sensors and Actuators A: Physical, vol. 194, pp. 176-180, May 2013.

[24] http://www.nemoto.eu/

[25] http://mipex-tech.com/en/infrared-sensors.html

[26] N. Samotaev, A. Vasiliev, A. Pisliakov, A. Sokolov, "Detection of smokeless pyrolysis of organic materials by metal, oxide gas sensor," Procedia Engineering, vol. 87, pp. 1322 - 1325, 2014.

[27] S. Bicelli et al., "Model and experimental characterization of the dynamic behavior of low-power carbon monoxide MOX sensors operated with pulsed temperature profiles," IEEE Trans. on Instrumentation and Measurement, vol. 58, no. 5, pp. 1324-1332, May 2009. 